\title{
A continuum viscous-elastic-brittle, Finite Element DG model for the fracture and drift of sea ice
}

\author{
Véronique Dansereau $^{1, *}$, Jérôme Weiss ${ }^{1}$, Pierre Saramito ${ }^{2}$ \\ 1 Institut des Sciences de la Terre (CNRS, UMR 5275), Université Grenoble Alpes, \\ 38058 Grenoble (France) \\ veronique.dansereau@univ-grenoble-alpes.fr \\ ${ }^{2}$ Laboratoire Jean Kuntzmann (CNRS, UMR 5224), Université Grenoble Alpes, 38058 Grenoble \\ (France)
}

\begin{abstract}
The thin ice covering the polar oceans is a complex geomaterial that is constantly breaking and moving under the action of the wind and ocean currents and that experiences transitions between a brittle solid and a granular fluid state. We have developed a simple continuum mechanical framework in the view of representing accurately its dynamical behavior in regional and global sea ice or coupled climate models. It combines the concepts of elastic memory, progressive damage and viscous-like relaxation of stresses. Here, we present this framework and its ongoing numerical development based on Finite Element, Discontinuous Galerkin methods.
\end{abstract}

Keywords: Solid-to-granular transition, brittle mechanics, strain localization, sea ice modeling, visco-elasto-brittle rheology, Discontinuous Galerkin methods

\section{Introduction}

From the continuum mechanics point of view, a number of geomaterials can be considered as both damageable elastic solids in which highly localized features emerge as a result of small deformations associated with brittle mechanisms and materials experiencing large, permanent ductile strains. Between these two behaviors, they also present transitions between a brittle solid and a granular fluid regime. Physically sound models of their mechanical and dynamical behavior must therefore represent both types of deformations, small and large, and the associated transitions between the different rheologies.

One important example is the Earth's crust, in which brittle fracturing and Coulomb stress redistribution are known to take place and for which scaling properties have been recognized for years (Turcotte, 1992 and others). Along active faults, co-seismic fracturing activates aseismic creep (Perfettini et al., 2010), leading to deformations that can be larger than those associated with the co-seismic fracturing itself (Cakir et al. 2012). Creep relaxes a significant amount of elastic strain, retarding stress accumulation along some portions of faults and concentrating stresses on other locked portions, a dissipa- 
tion process that should be included in earthquakes models (Gratier et al. 2014). In the case of volcanic edifices and landslides, small deformations associated with the fracturing and local crushing (Poli, 2017) of the bedrock cumulate and lead to much larger and potentially catastrophic deformations (Got et al., 2014; Riva et al., 2017).

Another example is sea ice - the thin, frozen layer that covers the polar oceans which readily fractures under the action of the wind and ocean drags. In this case, much larger deformations occur once cracks, called "leads", divide the brittle ice cover into ice plates, called "floes", which then move relative to each other with much reduced mechanical resistance. On the one hand, the resulting dynamical behavior of sea ice in a dense regime (where ice concentration is high with respect to unfrozen water: see Fig. 1, lower left panel), is known to be self-similar and strongly coupled in time and space, alike plate tectonics (Marsan and Weiss, 2010). Extensive scaling analyses of deformation data retrieved from ice-tethered buoys and Satellite Aperture Radar (Marsan et al. 2004; Hutchings et al., 2011; Weiss and Dansereau, 2017; Rampal et al., 2019) has established a multi-fractal heterogeneity and intermittency that holds over a range of time and space scales, to the temporal/spatial resolution of the data (hour/kilometer) to that of the winter season/Arctic basin. On the other hand, the sea ice cover can become highly fragmented and loose in some regions (see Fig. 1, upper left panel), due to either seasonal melt or intense fracturing by waves. In this granular-like regime, mechanical interactions are dominated by collisions between ice floes.

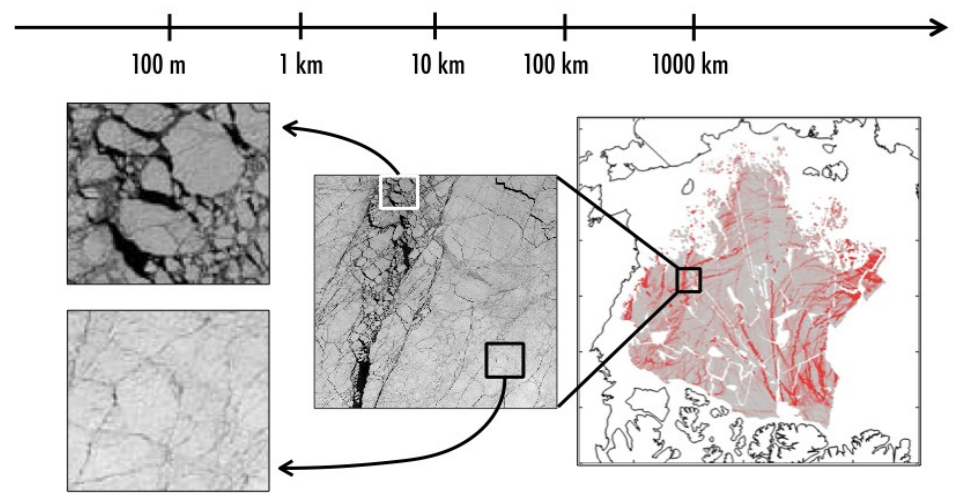

Figure 1. In the spatial domain, sea ice deformation is observed to be concentrated along linear features that are clearly identifiable over a wide range of space scales, up to that of the Arctic basin, as estimated via satellite imagery (right panel). Sea ice deformation also appears to be a self-similar, highly localized process in the time domain: isolated, short-duration fracturing events of various in tensities occur over a wide range of frequencies and sustain large-scale deformation events, maintaining the linear deformation features active for many days.

Both the small and large deformations associated respectively with fracturing and drift and the transitions between the dense and loose regimes must be accounted for in sea ice models, as they set the overall evolution of the ice cover. On the modeling point of view, representing the dynamics of sea ice therefore lies between a solid mechanics 
(small deformations) and a granular fluid dynamics (large deformations) problem. The capability to solve this problem in an accurate and numerically efficient manner is becoming especially important these days in the context of forecasting the opening of shipping routes, inferring the mechanical constraints on offshore structures and ships and estimating the future evolution of both its summer and winter extent.

While the more natural approach to cast a granular-type material would be the Discrete Element Method (DEM), a realistic DEM model of the Arctic or Antarctic sea ice cover for short and long-term predictions would entail simulating millions of ice floes of irregular shapes and wildly different sizes (Rothrock and Thorndike, 1984) as well as all of their mechanical interactions (collisions, friction). Such a model remains at present computationally too expensive. Most importantly, current DEM sea ice models lack the capability of representing the fracturing of multiple, realistic shape floes and become significantly more expansive as the frequency of interaction between floes increase, which makes them unsuitable for simulating sea ice in the dense regime.

Therefore, virtually all of the operational sea ice modeling platforms and climate models including a sea ice component that are currently in use are based on a continuum approach. In this framework, without a treatment of the kinematics of the discrete ice floes or individual leads, the relationship between the stress and the macroscopic deformation of the ice cover must be prescribed through a constitutive law. The biggest challenges of such approach are two-folds:

(1) the formulation of the constitutive law itself, which must allow representing the discontinuities that arise due to fracturing using continuous variables and gridcell averaged quantities. The temporal and spatial scaling properties highlighted by the aforementioned statistical analyses of sea ice deformation indeed indicate the absence of a characteristic time or space scale over the range of scales that are of interest in regional and global sea ice models (with a typical spatial resolution of a few to a hundred kilometers and a temporal resolution of hours to days). This implies that no space or time scale is suitable for defining average sea ice dynamics quantities (velocity fluctuations, deformation rates) over this range of scales and that sea ice deformation is also more localized at time and space scales below that of the model's temporal and spatial resolution. With this information in mind, perhaps the best a continuum sea ice model can do is to localize deformation at its smallest available (i.e., nominal) time and space scales, that is, the model time step and grid cell, and aim at reproducing accurately the observed scaling properties deformation at and beyond these scales.

(2) the implementation of numerical methods that must allow resolving and preserving the extreme gradients associated with discontinuities. This second challenge stems from the first one : a sea model that has the capability to localize deformation and associated fields at the nominal time and space scales while handling large deformations must inevitably employ numerical methods that limit the diffusion while advecting these fields.

Faced with these challenges, we have recently developed a continuum rheological model named Maxwell-elasto-brittle (MEB) destined for regional and global, stand- 
alone sea ice or coupled atmosphere-ocean-sea ice simulations. The mechanical framework combines the concepts of elastic memory, progressive damage mechanics, viscous-like relaxation of stresses and healing. Simulations of the sea ice cover have shown that it accurately reproduces the observed multi-fractal heterogeneity and intermittency of sea ice deformation, from its nominal time/space scales and beyond. The numerical framework is being developed on the basis of finite elements and Discontinuous Galerkin (DG) methods. Here, we present this continuum mechanical framework and our ongoing work on the use of DG methods with high-degree discrete polynomial approximations of the advection terms for the transported model variables.

\section{A Visco-Elasto-Brittle framework for the small and large deformations of sea ice}

\subsection{The MEB rheology}

The constitutive law of the MEB framework takes the form of the Maxwell viscoelastic model :

$$
\frac{1}{E} \frac{D \sigma}{D t}+\frac{1}{\eta} \boldsymbol{\sigma}=\boldsymbol{K}: \dot{\boldsymbol{\varepsilon}}
$$

with $E$, the elastic modulus, $\eta$, the viscosity, $\boldsymbol{\sigma}$, the stress tensor and $\phi(r)$, the rate of strain tensor. In the case of sea ice, it is applied to a compressible, elastic solid (hence the dimensionless elastic stiffness tensor, $\boldsymbol{K}$, defined in terms of the Poisson's ratio, 0 $\leq \nu<0.5$ ). The elastic component serves to represent the small, reversible deformation associated with brittle fracturing processes and the viscous component, the irreversible and potentially large deformation occurring once the ice cover is fractured (see Fig. 2). An important distinction with respect to the original Maxwell model is that the viscosity, $\eta$, associated with the stress dissipation term is not the bulk viscosity of the sea ice, but rather an "apparent" viscosity (Lyakovsky et al. 1997; Frederiksen \& Braun 2001 and others) that represents the resistance of the material to flow averaged over the model grid cell. Like the elastic modulus, $E$, this viscosity is allowed to evolve in both space and time according to the local level of damage of the material, i.e., its degree of fracturation/fragmentation. The ratio of the apparent viscosity and elastic modulus, $\lambda=\eta / E$, has the dimension of a time, and sets the rate of dissipation (or relaxation) of the stress through permanent deformations.

Based on previous isotropic progressive damage models, the level of damage is characterized by a scalar variable, $d$, varying between 0 for and undamaged and 1 for a "completely damaged" material. Consistent with in-situ stress measurements in sea ice (e.g., Weiss and Schulson, 2009), damage increases locally when the state of stress becomes overcritical with respect to a Mohr-Coulomb failure criterion and evolves at a rate related to the speed of propagation of elastic waves in the ice cover $(\sim 1000 \mathrm{~m} / \mathrm{s})$. 
Considering a typical spatial resolution between $1 \mathrm{~km}$ and $100 \mathrm{~km}$ for regional and global sea ice models respectively, this implies a characteristic damage propagation time scale, $t_{d}$, of a few seconds to a few hundred seconds. This propagation redistributes the stress from overcritical to subcritical areas, thereby triggering avalanches of damaging events and the formation of macroscopic leads (cracks). To account for healing processes that counterbalances the effects of damaging over much longer time scales, the level of damage is also allowed to decrease at a rate controlled by a characteric healing time scale, $t_{d}$. In the context of sea ice, this represents the refreezing of water within open leads, which typically takes a few days $\left(10^{5}\right.$ to $\left.10^{6} \mathrm{~s}\right)$. Healing and damaging are combined in the following evolution equation for the level of damage:

$$
\frac{D d}{D t}=\left(1-\min \left[1, \frac{\sigma_{c}}{\sigma_{1}-q \sigma_{2}}\right]\right)(1-d) \frac{t}{t_{d}}-\frac{t}{t_{h}}, 0 \leq d<1,
$$

where $\sigma_{l}$ and $\sigma_{2}$ are the principal stresses, $q$ is the slope of the Mohr-Coulomb criterion in the $\left(\sigma_{1}, \sigma_{2}\right)$ space and $\sigma_{c}$ is the uniaxial (unconfined) compressive strength of sea ice.

The coupling between both $E$ and $\eta$ and $d$, represented in Fig. 2, is such that over undamaged areas, the elastic modulus is maximal and the apparent viscosity, infinitely large. In this limit, the mechanical response is strictly elastic and the resulting small and highly localized deformations are associated with fracturing processes. Conversely, over highly fragmented areas, both $E$ and $\eta$ decrease, which allows the dissipation of part of the stress into permanent and potentially large deformations. Because the rate of dissipation of the stress through permanent deformations also decreases as the ice cover becomes more fragmented, the relaxation time, $\lambda=\eta / E$, must decrease with increasing $d$. In order to meet this criteria with the simplest possible function while considering the definition of the effective elastic stress in the theory of progressive damage, which implies $E=E_{0}(1-\mathrm{d})\left(\right.$ Kachanov, 1958), we set $\eta=\eta_{0}(1-\mathrm{d})^{\alpha}$, hence $\lambda=\lambda_{0}(1-\mathrm{d})^{\alpha-1}$, where $E_{0}, \eta_{0}$ and $\lambda_{0}$, are the undamaged mechanical parameter values and $\alpha>1$, a constant exponent. The value of $\alpha$ is constrained through sensitivity analyses with the goal of reproducing the scaling properties of sea ice (Weiss and Dansereau, 2017).
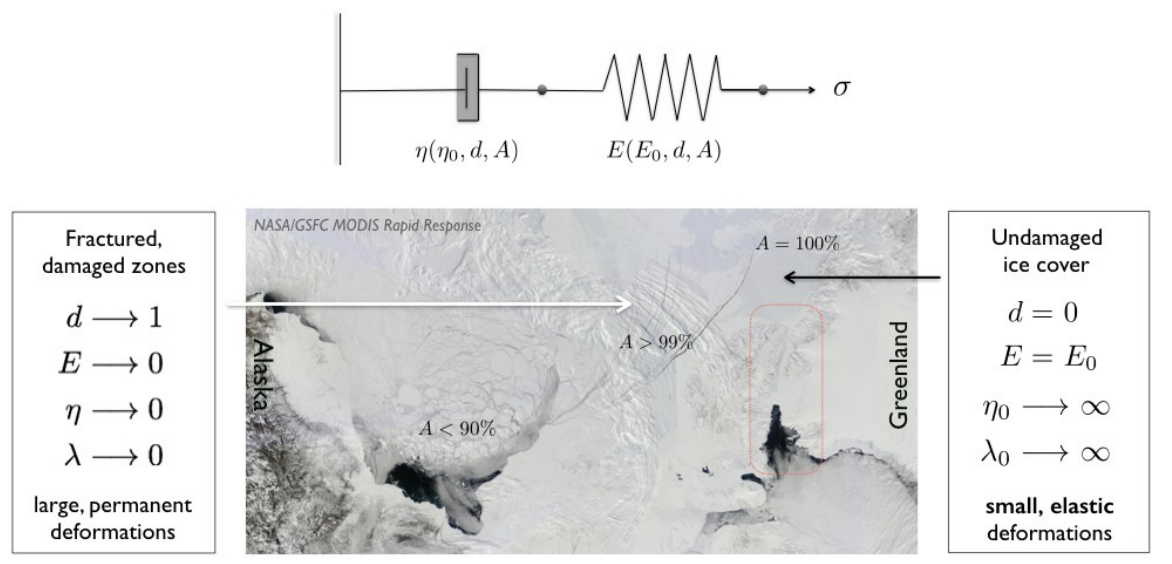
Figure 2. MODIS image of the sea ice over the Arctic Canadian Basin on 07/05/2015, showing high concentration, fractured and unfractured, zones as well as low concentration zones and illustrating the dependence of the apparent viscosity, $\eta$, elastic modulus, $E$, and relaxation time, $\lambda$, on the level of damage in the MEB rheology (https://worldview.earthdata.nasa.gov/).

\subsection{Implementation in the context of sea ice modeling}

In the MEB rheology, the elastic modulus and apparent viscosity are allowed to depend on an another critical property : the concentration of ice over the model grid cell, A. Similar to the packing fraction for a granular material, this variable evolves between 0 for a water-covered cell and 1 for a sea ice-covered cell. Its coupling to the mechanical parameters follows the exponential function suggested by Hibler (1979)

$$
\begin{aligned}
& \eta=\eta_{0}(1-d)^{\alpha} \exp [-c(1-A)], \\
& E=E_{0}(1-d) \exp [-c(1-A)],
\end{aligned}
$$

where $\mathrm{c}$ is a non-dimensional constant and which allows $E$ and $\eta$ to be maximal when the ice concentration is $100 \%(A=1)$ and to decrease rapidly when leads open and $A$ drops ( $\sim 10 \%$ at $A=90 \%$, see Fig. 2). In the case of $\eta$, this parametrization is compatible with the rapid decay of the apparent viscosity of granular media when decreasing their packing fraction from the close-packed limit (Aranson and Tsimring, 2006).

The sea ice cover is typically a few meters thick and millions of km square in area. Hence in sea ice models, it is represented as a two-dimensional plate with plane stresses. Its motion is described by the following Navier-Stokes type equation:

$$
\rho h \frac{D \boldsymbol{u}}{D t}=A\left(\boldsymbol{\tau}_{\boldsymbol{a}}-\boldsymbol{\tau}_{w}\right)-\boldsymbol{f}_{\text {Coriolis }}-\boldsymbol{f}_{S S H}+\nabla \cdot(h \boldsymbol{\sigma}) .
$$

with $\boldsymbol{u}$, the ice velocity, $\rho$, the ice density, $\boldsymbol{\tau}_{a}$ and $\boldsymbol{\tau}_{w}$, the air and water drags, $\boldsymbol{f}_{\text {Coriolis }}$, the Coriolis pseudo-force, and $\boldsymbol{f}_{S S H}$, the force due to gradients in the sea surface dynamic height. The variable $h$ represents the mean ice thickness over a model grid cell. Mass conservation is ensured by the following evolution equations for $h$ and $A$ :

$$
\begin{gathered}
\frac{D A}{D t}+A \nabla \cdot \boldsymbol{u}=T_{A}, 0 \leq A \leq 1,, \\
\frac{D h_{\text {thick }}}{D t}=T_{h},
\end{gathered}
$$

where $h_{\text {thick }}=h / A$ is the mean thickness of the ice-covered portion of a grid cell and $T_{A}$ and $T_{h}$ represent thermodynamic source/sink terms of $A$ and $h$ (i.e., ice growth/melt). Ice can also pile-up where motion is convergent. This is represented by capping $A$ to 1 and redistributing the excess ice concentration over a grid cell by increasing $h$. Redistribution schemes of different levels of complexity exist, but almost all are based on a constant ice density and conservation of ice volume. 
Very simple simulations representing a uniaxial compression experiment without advection, with an idealized domain geometry and homogeneous forcing conditions, have shown that the MEB rheology, with few independent variables, can represent a large range of mechanical behaviors (Dansereau et al. 2016; Weiss \& Dansereau 2016). In particular, it reproduces both the persistence of creeping faults/leads, the activation of new faults/leads with different shapes and orientations, temporal correlations in the rate of damage at all timescales and spatial localization down to the nominal space scale.

The MEB rheology has also recently been implemented in NeXtSIM (Bouillon and Rampal, 2015), a continuum sea ice model developed at the Nansen Environmental and Remote Sensing Center (Norway) for operational forecasting and climate prediction purposes. This Lagrangian, Finite Element model uses reanalysis ocean and atmospheric forcings and include thermodynamic processes, allowing to perform realistic and longterm simulations of the Arctic sea ice cover (see Figs. 3a, b) and thereby validate the model against observations. A statistical analysis of the fields of deformation simulated by NeXtSIM (Rampal et al., 2019) has shown that the model reproduces well the observed spatial and temporal scalings of sea ice deformation and, in particular, their multi-fractality (see Fig. 3b). The simulated scalings hold over a wide range of space and time scales, down to the grid cell size and almost to the model time step.
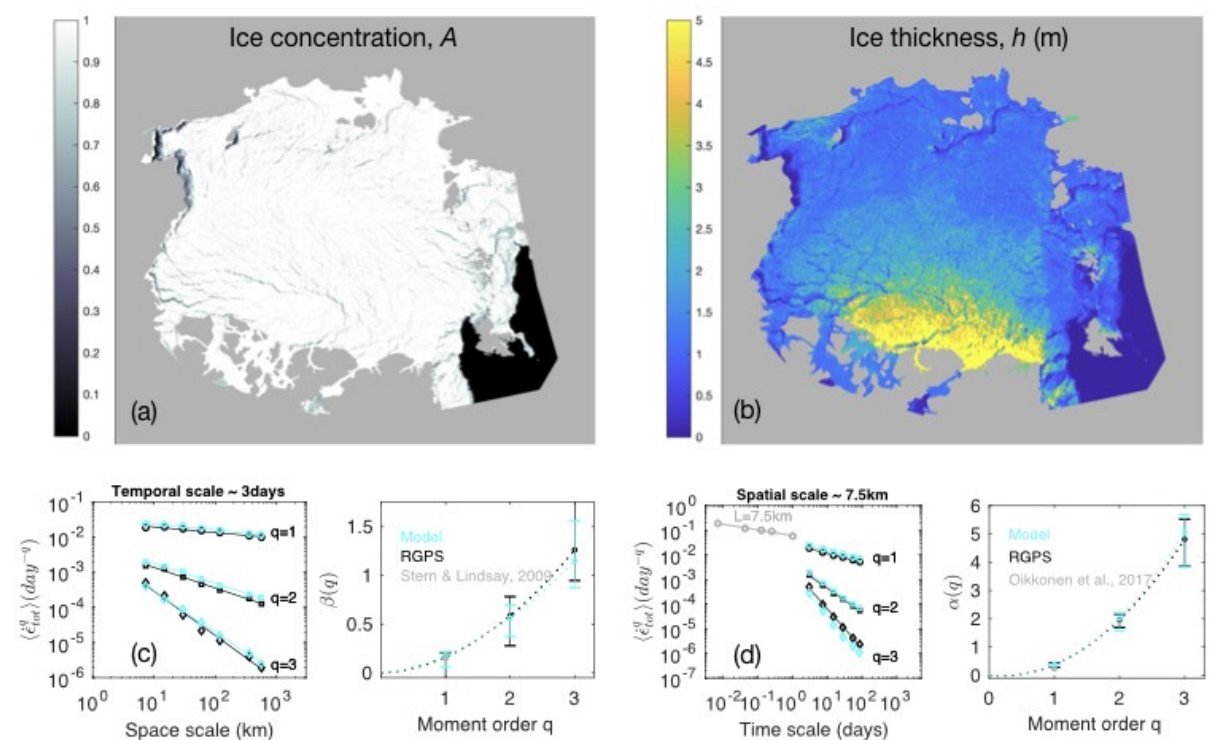

Figure 3. Field of (a) sea ice concentration and (b) thickness simulated with the NeXtSIM model. Reproduced from Rampal et al., 2019: (c) Spatial and (d) temporal scaling analyses of the total deformation rate simulated by NeXtSIM (cyan curves) and derived from satellite observations (black curves). The scalings are calculated for the moments of order 1, 2 and 3 of the distribution of the total deformation rate, at (c) a temporal scale of 3 days and space scales varying from 7.5 to $580 \mathrm{~km}$ and (d) a spatial scale of $7.5 \mathrm{~km}$ and time scales varying from 3 to 100 days. The corresponding structure functions support multifractal scalings for both the model and satellite observations. 


\section{Treating large deformations}

These cited studies have demonstrated the capability of the MEB rheology to localize deformation at the nominal model time and space scales, whatever the scale. Consequently, the numerical framework on which a MEB sea ice model is build is key to its development, as it must allow preserving this localization when advection is represented. In building the MEB framework, we choose to take advantage of the recent expansion of finite element (FE) methods in the fields of climate modeling. This choice leaves the option of a Eulerian, Lagrangian or mixed framework.

While the most common approach to model the deformation of continuum solids is to cast the equations of motion in Lagrangian form, most sea ice codes use the Eulerian form instead. As these are based on a viscous-plastic sea ice rheology (Hibler, 1979; Hunke and Dukovicz, 1997), they are indeed compatible with a fluid-type approach and by the same fact straightforwardly coupled with ocean and atmospheric models (which are virtually all Eulerian). One exception in the sea ice community is the aforementioned NeXtSIM model (see section 2.2), which uses a Lagrangian framew ork and a dynamical remeshing scheme (Samake et al., 2017). Adaptive remeshing techniques are becoming more and more computationally efficient. However, remeshing inevitably entails some numerical diffusion associated with the repeated interpolation of the modeled quantities onto restructured meshes, which is hard to quantify. It can introduce some inaccuracy in the model solution, as the restructured fields might not respect the local force balance or yield criterion. Also, coupling a Lagrangian sea ice model to Eulerian atmosphere and ocean models requires interpolating the shared variables onto the fixed ocean/atmosphere model meshes, thereby inducing some interpolation-related diffusion and a loss of localization. The Material Point Method (Sulsky et al., 1994, 1995), developed to efficiently handle large deformations and the transport of material properties in continuum models, was also implemented in the context of sea ice modeling by Sulsky et al. (2007). However, a general limitation of this approach is that for certain configurations of the flow, for instance under local divergence or convergence, some grid elements can be either left empty of material points or significantly over-represented. The density of points then need to be made more uniform over the computational domain by somehow splitting, merging, creating or destroying points. In the case of sea ice, this can be partially dealt with through thermodynamics considerations: material points can be created through refreezing within leads and destroyed where the ice melts (Sulsky et al., 2007). Nonetheless, it necessitates the implementation of possibly non-trivial and/or non-physical artifacts.

With the idea of taking the path that is the most compatible with the numerical habits of the climate modeling community while preparing for a coupling of an MEB-based sea ice model with an atmosphere and ocean component, we decided to cast the equations of motion in the Eulerian frame and to write their variational formulation using discontinuous Galerkin methods. These methods, which can be viewed as a higher order upwind finite volume scheme in which the solution is approximated over each mesh element as a polynomial function instead of being piecewise constant, have experienced a 
significant expansion in recent years, including in the fields of atmosphere and ocean modeling, owing to their numerous advantages. They are known to be (i) very stable and well suited for problems where advection is dominant, (ii) efficient for discontinuous approximation of stress tensors in viscoelastic flow problems (Saramito, 2014) and (iii) readily parallelizable, which in the context of modelling sea ice at high-resolution on large scales, is a highly valuable property. To our knowledge, such methods have not yet been employed in a sea ice model based on an elastic rheology nor in a viscous-elastic-brittle model in general. This makes the implementation and testing of discontinuous Galerkin methods within the MEB framework an interesting experiment in itself.

\subsection{Tests on advection and diffusion : rigid body rotation}

We first evaluate the capability of a discontinuous Galerkin-based numerical scheme in handling the advection of fields showing very strong gradients, as that of $\boldsymbol{u}, \boldsymbol{\sigma}, A, h$ and $d$ generated by the MEB model. We do so by comparing the analytical and numerical solutions of a rigid body rotation problem, the configuration of which is shown in Fig. 4a. The diffusivity of the numerical scheme is analyzed here as a function of the order of the finite element approximations. An extended analysis including the sensitivity of the solution to the time and space resolutions as well as to the order of the temporal discretization scheme can be found in Dansereau, $\mathrm{PhD}$ thesis (2016).

The 2-dimensional domain, $\Omega$, represents a circular, rigid disc that is rotated by prescribing a constant angular frequency, $\omega$ (see Fig. 4a). The velocity, $\boldsymbol{u}(r, \theta)$, is given everywhere by $\boldsymbol{u}(t)=-\omega \boldsymbol{r} \boldsymbol{e}_{\theta}$, with

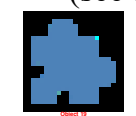

. We consider a passive scalar field,

Eq. 8 is discretized in time using a Euler implicit scheme. The domain, $\Omega$, is discretized in space using a two-dimensional mesh, $T_{h}$, with triangular elements (see Fig. 4a). The transport equation is written in weak form by introducing a scalar test function, $\varphi_{h}$, for the field $\phi$ and the discontinuous finite element space $S_{h}=\left\{\varphi_{h} \in L^{2}(\Omega) ; \varphi_{h \mid K} \in P_{k}, \forall\right.$ $K \in T_{h}$ \}, with discontinuous elements $P_{k}$ of degree $k \geq 0$. The code is developed within the C++ environment RHEOLEF (Saramito, 2013).

We compare the accuracy of the numerical scheme between three fields of $\phi$ with different levels of regularity: $\phi_{1}$ is a cosine function, $\phi_{2}$ is a locally discontinuous step function and $\phi_{3}$ a field of uncorrelated noise, defined over each model element by drawing randomly the value of $\phi$ from a uniform distribution (see Fig. 4a). The first function represents the regularity of the fields that would be obtained with a model that either could not localize deformation and associated variables at the nominal spatial scale or would use a regularization method such as non-local damage. The regularity of the fields simulated by the MEB model, which are characterized by the presence of 
highly localized linear features, can instead be considered to lie somewhere between that of the second and third fields of $\phi$. Indeed, as these linear features introduce spatial correlations, at least in one dimension, $d, A$ and $h$ are more regular than pure uncorrelated noise, but, due to the tendency of the model to localize deformation at the scale of the element, they show more discontinuities than a linear step function. Diffusion of the strong gradients within these fields is therefore expected to be less important than for $\phi_{3}$, but more than for $\phi_{1}$ and $\phi_{2}$.

Fig. 4 shows the initial fields of $\phi$ and gives a tastes of the amount of diffusion in the model by comparing the initial values of $\phi(r)$ as a function of $\theta$ with the values after one quarter of a revolution and complete revolution of the rigid disc, in the case of polynomial approximations of degree $0\left(P_{0}\right), 1\left(P_{1 d}\right)$ and $2\left(P_{2 d}\right)$. Based on the work of Dansereau, $\mathrm{PhD}$ thesis (2016), the order of the time discretization scheme is respectively of 1,2 and 3 in the $P_{0}, P_{1 d}$ and $P_{2 d}$ cases.
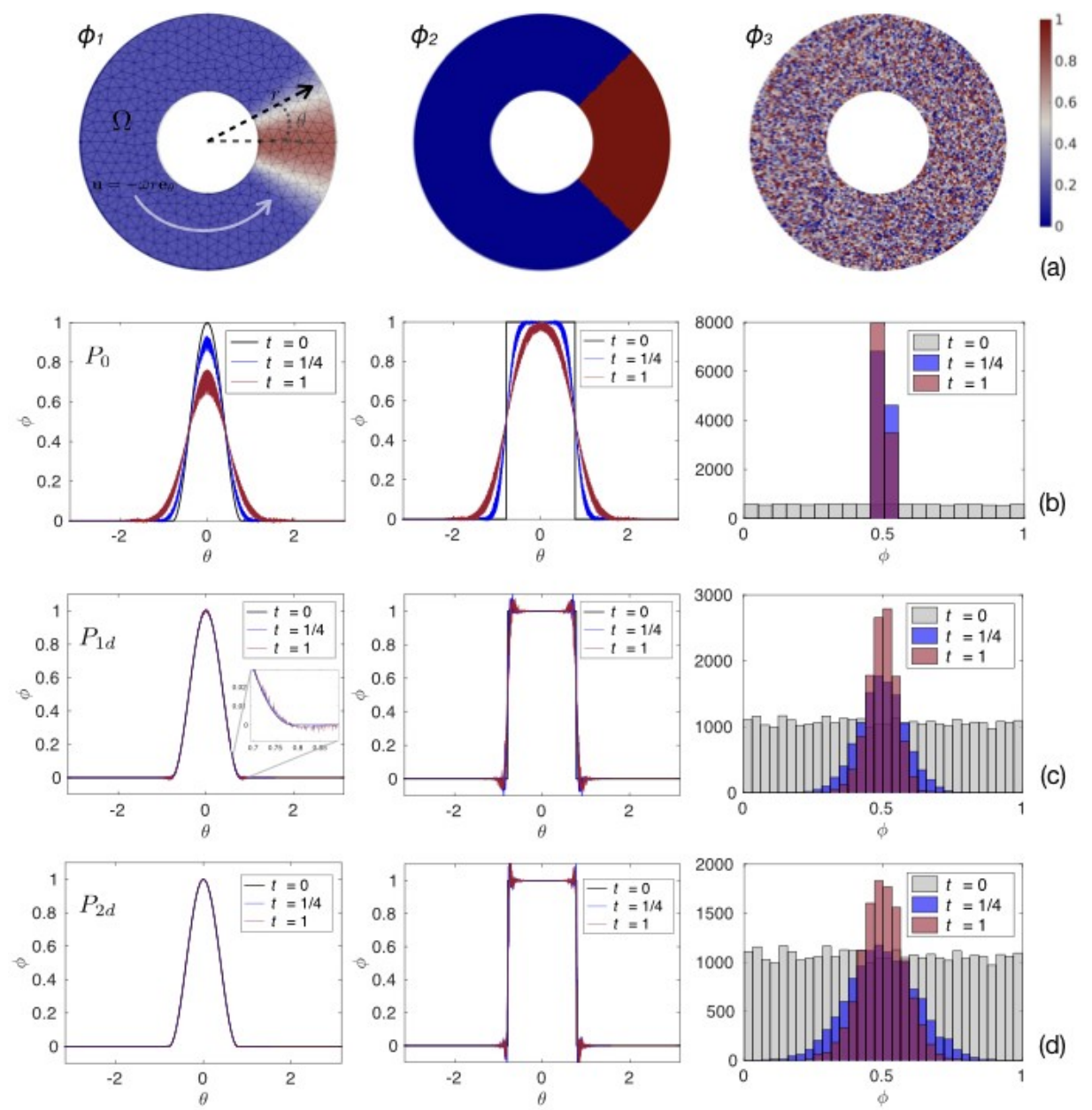
Figure 4. (a) Fields of $\phi_{1}, \phi_{2}$ and $\phi_{3}$ at $t=0$ interpolated onto $T_{h}$ using FE approximations of order $k$ $=0$. The upper left panel shows the (adimensional) domain, $\Omega$, and an example of mesh, $T_{h}$. The actual temporal and spatial resolutions for the experiments are $1 / 10000$ and $1 / 40$. (b) Corresponding $P_{0}$ approximation of the fields of $\phi_{1}$ and $\phi_{2}$ and distribution of $\phi_{3}$ at $t=0$ (black curve), $t=1 / 4$ (blue curves) and $t=1$ (red curves). Corresponding (c) $P_{l d}$ and (d) $P_{2 d}$ discontinuous approximations.

For $P_{0}$ approximations, the fields of $\phi$ are constant by element, and the DG scheme coincides with a finite volume approximation with upwinding, which is known to be highly diffusive. The most regular function, $\phi_{1}$, indeed shows a difference in amplitude of the initial and final approximations between $25 \%$ and $35 \%$ (Fig. 4b). The step function, $\phi_{2}$, looks like a smooth sinusoid after one complete revolution. In the case of the field of uncorrelated noise, as we are not interested in its specific spatial structure, $\phi_{3}$, we instead evaluate the numerical diffusion based on the evolution of its distribution. Fig. $4 \mathrm{~b}$ shows that after $1 / 4$ of a revolution, it is almost completely homogenized.

For the most regular function, $\phi_{1}$, increasing the order of the FE approximations by 1 degree seems sufficient to practically eliminate numerical diffusion (Fig. 4c). For the step function $\phi_{2}$, the numerical scheme almost perfectly preserves the sharp gradients around the initial location of the discontinuities for $P_{2 d}$ approximations (Fig. 4d). Increasing the order of the approximations also reduce the rate at which the field of noise, $\phi_{3}$, homogenizes. One important observation however is that in the case of an implicit time discretization scheme, the DG method results in non-monotonic dispersion and generates values of $\phi$ outside of the interval over which it is initially defined. This effect is more important around local discontinuities and as the advected gradients are strong (compare for instance Figs. $4 \mathrm{c}$ and $4 \mathrm{~d}$ for $\phi_{2}$ and $\phi_{3}$ around $\theta= \pm \pi / 4$ ). Yet, in the MEB framework, some of the advected fields must evolve within a certain range of values, for instance the level of damage $d$, defined on the interval $[0,1]$. While this problem can be solved by simply constraining manually such variable to lie within a prescribed interval, the generation of nonphysical values due to spurious oscillations of the solution could also be avoided by replacing the implicit time discretization scheme used here by a Strong Stability-Preserving Runge-Kutta scheme (Gottlieb et al., 2008) for which the dispersion of the DG method is known to be strictly monotonic (e.g., Hanert et al., 2004). This development is now being studied.

These simple tests suggest that DG methods could be efficient in handling the advection of the strong gradients generated by the MEB model, provided that the order of the FE element approximations for the advected fields can be of order $\mathrm{k}>0$.

\subsection{Tests on advection and diffusion: channel simulations}

As a second evaluation of the robustness of DG methods in handling the advection of the extreme gradients simulated by the MEB model, we conduct simulations of the flow of sea ice into a channel (see Fig. 5) with dimensions that are representative of the numerous channels of the Canadian Arctic Archipelago (CAA), through which significant volumes of sea ice are exported out of the Arctic Basin under the action of the winds. In 
a manner similar to granular cohesive materials that form concave stress-free surfaces and exhibit self-obstruction to flow under certain conditions (e.g., sand or grain in a silo, Richmond and Gardner; 1962; Walker, 1966), the CAA is known for the formation of sea ice "bridges" (Kwok et al., 2010; Münchow, 2016); curved and concave arches that can remain stable and block the flow of ice in channels for weeks to months (see Fig. 2, red box, for an example). Once a stable ice bridge forms, the ice downstream detaches from it and is driven out of the channel without mechanical resistance. At this point, internal stresses drop and no further fracturing is expected in the detached part, which is drifting freely and in which the diffusion of gradients is therefore expected to be maximum. In the part of the channel upstream of the bridge, stresses continue to increase as ice piles up while being pushed by the wind. Extremely sharp gradients in ice velocity, thickness and concentration thus arise between the upstream and downstream portions of the channel, which makes these simulations a severe benchmark for testing the capability of the numerical scheme in handling both discontinuities and advection.

Eqs. 1-7 are solved here, without thermodynamics, an ocean at rest and the thickness redistribution defined as in Dansereau et al., 2017. All simulations are started from rest with a uniform, $100 \%$ concentration, undamaged and $1 \mathrm{~m}$ thick ice cover, so that the location of ice bridges is not prescribed. A no-slip condition is applied at the coasts and the channel is open at its top and bottom boundaries (see Fig. 5), where the value of all transported quantities is prescribed so that represent undamaged ice entering the channel ( $d=1, A=1, h=1 \mathrm{~m}$ and $\boldsymbol{\sigma}=0)$. An along-channel, southward, and spatially uniform wind stress, $\boldsymbol{\tau}_{a}$, is applied. It is increased steadily between 0 and $1 \mathrm{Nm}^{-2}$ over a period of 24 hours and then held constant to simulate the passage of a storm. All of the advection and non-linear terms in the material derivatives of Eqs. 1, 2, 5-7 are solved using DG methods. The unstructured mesh with triangular elements has an average spatial resolution of $4 \mathrm{~km}$ and the time step is $8 \mathrm{~s}$. The numerical scheme is described in details in Dansereau et al. (2017).
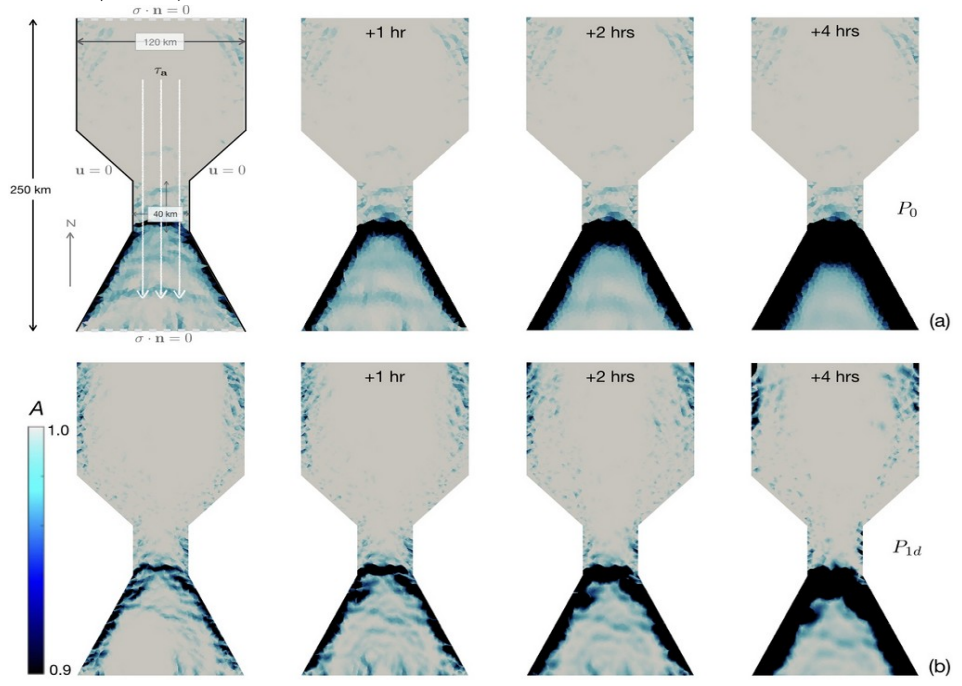
Figure 5. Simulations of sea ice flow in a channel for (a) $P_{0}$ and (b) $P_{l d}$ polynomial approximations of the advected fields. The upper left panel shows the domain, boundary and forcing conditions. The leftmost panels show the fields of ice concentration at the moment the ice downstream detaches from the ice bridge. The other panels show the ice concentration 1, 2 and 4 hours later, when the detached ice is drifting out of the domain.

Fig. 5 compares the simulated fields of ice concentration between simulations using respectively $P_{0}$ and $P_{1 d}$ approximations for the advected fields and $P_{1}$ and $P_{2}$ continuous approximation for the velocity field. In both cases, a concave ice bridge form and the model reproduces the very sharp gradients in ice concentration, thickness and velocity associated with the edge of the ice bridge while remaining numerically stable. Dansereau et al. 2017, have shown that this is also the case in realistic channel simulations. As expected, numerical diffusion is most important at the edge and within the detached ice plate, where mechanical stresses vanish and drift velocities are the highest. One hour after the detachment of the ice from the bridge, gradients associated with the presence of cracks in the detached plate are significantly diffused in the the $P_{0}$ case. After 2 and 4 hours, the edge of the detached plate also appears completely smoothed. A calculation of the volume of the detached plate would probably reveal a significant volume loss associated with the diffusion of its edge. Comparatively, increasing the order of the polynomial approximation by 1 degree significantly reduces the smearing of the gradients within the detached plate and conserves the irregular shape of its edge. In both simulations, where ice concentration is high and drift velocities are low, such as upstream of the bridge, mechanical stresses are significant and constantly redistributed by damage events. The associated deformation is extremely localized in both space and time, which conserves extreme gradients in all fields. However, where ice concentration is low and drift velocities high, increasing further the order of polynomial approximations would be necessary to conserve extreme gradients in the context of longer term simulations, albeit the increased computational times. Such simulations are underway.

\section{Conclusions}

In this paper, we have presented a simple rheological framework that is capable of representing both the small and large deformations of the sea ice cover and its extreme temporal and spatial localization. The model was also shown to simulate a mechanical behavior that is compatible with a fragmented sea ice cover and a granular-type rheology, such as the formation of stable concave ice bridge that block the flow of ice in narrow channels. Considering that all existing numerical frameworks, Lagrangian, Eulerian or mixed, imply some amount of diffusion that can only be reduced at a numerical cost, we have decided on an Eulerian framework, which presents the advantage of being easily coupled in the context of future ocean-atmosphere-sea ice simulations. We have based our approach on Discontinuous Galerkin methods, which appear to be a skillful solution for polynomial approximation of order $>0$. To determine the minimal order of 
the polynomial approximations required in the context of long-term sea ice simulations, we plan to perform realistic simulation of the Arctic sea ice that would allow comparing the model fields with observations and verify if the simulated sea ice deformations exhibits the observed degree of spatial and temporal localization, with the associated multi-fractal scalings.

Acknowledgements Part of this work has been financially supported by TOTAL EP RECHERCHE DEVELOPPEMENT. The authors thank the sea ice modeling team at the Nansen Environmental and Remote Sensing Center for sharing the results of NeXtSIM simulations.

\section{References}

Aranson, I. S. and Tsimring, L. S. 2006. Patterns and collective behavior in granular media: Theoretical concepts. Reviews of Modern Physics, 78, 641-692.

Bouillon, S., and Rampal, P. (2015). Presentation of the dynamical core of neXtSIM a new sea ice model. Ocean Modelling, 91(0), 23-37.

Cakir, Z., Ergintav, S., Ozener, H., Dogan, U., Akoglu, A. M., Meghraoui, M. and Reilinger, R. (2012). Onset of aseismic creep on major strike-slip faults. Geology, 40(12), 1115-1118.

Dansereau, V. (2016) A Maxwell Elasto-Brittle model for the drift and deformation of sea ice, Ph.D. thesis, Université Grenoble-Alpes, 136 pages.

Dansereau, V., Weiss, J., Saramito, P., and Lattes, P. (2016). A Maxwell elasto-brittle rheology for sea ice modelling, The Cryosphere, 10, 1339-1359.

Dansereau, V., Weiss, J., Saramito, P., Lattes, P., and Coche, E. (2017). Ice bridges and ridges in the Maxwell-EB sea ice rheology, The Cryosphere, 11, 2033-2058.

Gottlieb, S., C.-W. Shu, and E. Tadmor. (2008). Strong stability-preserving high-order time discretization methods. Society for Industrial and Applied Mathematics, 43(1), 89-112.

Kagan, Y. Y., and Knopoff, L. (1980). Spatial distribution of earthquakes: the two-point correlation function. Geophysical Journal International, 62(2), 303-320 .

Got, J.-L., Carrier, A., Marsan, D., Jouanne, F., Vogfjörd, K., Villemin, T. 2017. An analysis of the non-linear magma-edifice coupling at Grimsvötn volcano (Iceland), Journal of Geophysical Research Solid Earth, 122, 10.1002/2016JB012905.

Gratier, J. P., Renard, F. and Vial, B. (2014). Postseismic pressure solution creep: Evidence and time-dependent change from dynamic indenting experiments. Journal of Geophysical Research: Solid Earth, 119(4), 2764- 2779.

Hanert, E., Le Roux, D. Y., Legat, V., and Deleersnijder, E. (2004). Advection schemes for unstructured grid ocean modelling. Ocean Modelling, 7, 39-58. 
Hibler, W. D. I. (1979). A dynamic thermodynamic sea ice model. Journal of Physical Oceanography, 9(7), 815-846.

Hunke, E., and Dukovicz, J. (1997). An elastic-viscous-plastic model for sea ice dynamics. Journal of Physical Oceanography, 27, 1849-1867.

Hutchings, J. K., Roberts, A., Geiger, C. A., and Richter-Menge, J. (2011). Spatial and temporal characterization of sea-ice deformation, Ann. Glaciol., 52, 360-368.

Kachanov, L. M. (1958), On time to rupture in creep conditions (in russian), Izviestia Akademii Nauk SSSR, Otdelenie Tekhnicheskikh Nauk, 8, 26-31.

Kwok, R., L. Pedersen, P. Gudmandsen, and S. Pang, 2010. (2007). Large sea ice outflow into the nares strait in 2007. Geophysical Research Letters, 37, L03, 502.

Marsan, D. and Weiss, J. (2010). Space/time coupling in brittle deformation at geophysical scales, Earth Planet. Sci. Lett., 296, 353-359.

Marsan, D., Stern, H. L., Lindsay, R., and Weiss, J. (2004). Scale dependence and localization of the deformation of Arctic sea ice, Phys. Rev. Lett., 93, 178501.

Oikkonen, A., Haapala, J., Lensu, M., Karvonen, J., and Itkin, P. (2017). Small-scale sea ice deformation during N-ICE2015: From compact pack ice to marginal ice zone, J. Geophys. Res.Oceans, 122, 5105-5120.Perfettini, H., Avouac, J., Tavera, H. et al. (2010). Seismic and aseismic slip on the Central Peru megathrust. Nature 465, 78-81.

Poli, P. (2017). Creep and slip: Seismic precursors to the Nuugaatsiaq landslide (Greenland), Geophys. Res. Lett., 44, 8832- 8836.

Rampal, P., Dansereau, V., Olason, E., Bouillon, S., Williams, T., Korosov, A., and Samaké, A. (2019). On the multi-fractal scaling properties of sea ice deformation, The Cryosphere, 13, 24572474

Riva, F., Agliardi, F., Amitrano, D., and Crosta, G. B. (2018). Damage-based time-dependent modeling of paraglacial to postglacial progressive failure of large rock slopes. Journal of Geophysical Research: Earth Surface, 123, 124- 141.

Richmond, O., and G. Gardner (1962): Limiting spans for arching of bulk materials in vertical channels. Chemical Engineering Science, 17(12), 1071 - 1078.

Samake, A., Rampal, P., Bouillon, S., and Olason, E. (2017). Parallel implementation of a Lagrangian-based model on an adaptive mesh in $\mathrm{C}++$ : Application to sea-ice. Journal of Computational Physics, 350, 84-96.

Saramito, P. (2013). Efficient C++ finite element computing with Rheolef: vol. 2. discontinuous Galerkin methods, CNRS-CCSD ed.

Saramito, P. (2014). On a modified non-singular log-conformation formulation for Johnson-Segalman viscoelastic fluids. Journal of Non-Newtonian Fluid Mechanics, 211, 16-30. 
Sulsky, D., Chen , Z., and Schreyer, H. L. (1994). A particle method for history-dependent materials. Computer Methods in Applied Mechanics and Engineering, 118(12), 179 - 196.

Sulsky, D., Zhou, S.-J., and Schreyer, H. L. (1995). Application of a particle-in-cell method to solid mechanics. Computer Physics Communications, 87(12), 236 - 252.

Sulsky, D., Schreyer, H., Peterson, K., Kwok, R., and Coon, M. (2007). Using the material-point method to model sea ice dynamics. Journal of Geophysical Research, 112, C02S90.

Rothrock, D. A. and Thorndike, A. S. (1984). Measuring the sea ice floe size distribution, J. Geophys. Res.-Oceans, 89, 6477-6486.

Turcotte, D. L. (1992). Fractals and Chaos in geology and geophysics. Cambridge University Press, New York, NY, USA.

Walker, D. (1966) An approximate theory for pressures and arching in hoppers. Chemical Engineering Science, 21(11), 975 - 997.

Weiss, J., and Schulson, E. M. (2009). Coulombic faulting from the grain scale to the geophysical scale: lessons from ice. Journal of Physics D: Applied Physics, 42(21), 214 - 227.

Weiss, J. (2017). Exploring the "solid turbulence" of sea ice dynamics down to unprecedented small scales, J. Geophys. Res.-Oceans,122, 6071-6075.

Weiss, J., and Dansereau, V. (2017) Linking scales in sea ice mechanics, Philos. Trans. Roy. Soc. A, 375, 20150352. 